\title{
Bone Maturation and Mineralization in Precocious Puberty
}

\author{
Yuko Nakayama, Osamu Arisaka, Atsuto Hosaka and Keijiro Yabuta \\ Department of Pediatrics, Juntendo University School of Medicine, Tokyo, Japan
}

Key words: precocious puberty, bone age, bone mineral density, lumbar spine, femoral neck

Introduction

Sex steroids and growth hormone (GH) are determinants of bone mineral density (BMD), and sex steroids in particular are thought to play an important role in increasing BMD during puberty (1), as well as promoting bone maturation. To assess the relationship between bone maturation and bone mineralization during exposure to excess sex steroids, we measured BMD in patients with early pubertal development associated with advanced bone age (BA).

\section{Subjects and Methods}

\section{Patients}

Ten children with central precocious puberty and one with premature pubarche were studied. They comprised 2 boys and 9 girls, aged 6-15 years. One of the boys and 4 of the girls had been treated with subcutaneous or nasal LH-RH analogue, and the remaining 6 patients were untreated.

Correspondence: Dr. Yuko Nakayama, Department of Pediatrics, Juntendo University School of Medicine, 3-1-3 Hongo, Bunkyo-ku, Tokyo 113 Japan

\section{Methods}

Height and weight were measured, and sexual maturity assessed by a single investigator using the criteria of Tanner. Bone age was analysed using the TW2 RUS method for Japanese children. Sex steroids and insulinlike growth factor-I (IGF-I) were measured using commercially available radoimmunoassay kits.

BMD $\left(\mathrm{gm} / \mathrm{cm}^{2}\right)$ was assessed by dual energy X-ray absorptiometry (Hologic QDR$2,000)$ at the lumbar vertebrae (L2-4) and right femoral neck.

\section{Results}

All the patients showed accelerated bone age for chronological age (CA) (BA/CA:1.091.62), and elevated serum concentrations of sex steroids and IGF-I. Pubertal development ranged from Tanner stages II to $\mathrm{V}$.

Values of BMD for the spine exceeded the normal mean for CA in 7 of the 11 patients. However, BMD values adjusted to BA were below the normal mean in both sexes (Fig. 1, Fig. 2). BMD for the right femoral neck was 
slightly lower than for the lumbar spine except in 2 cases.

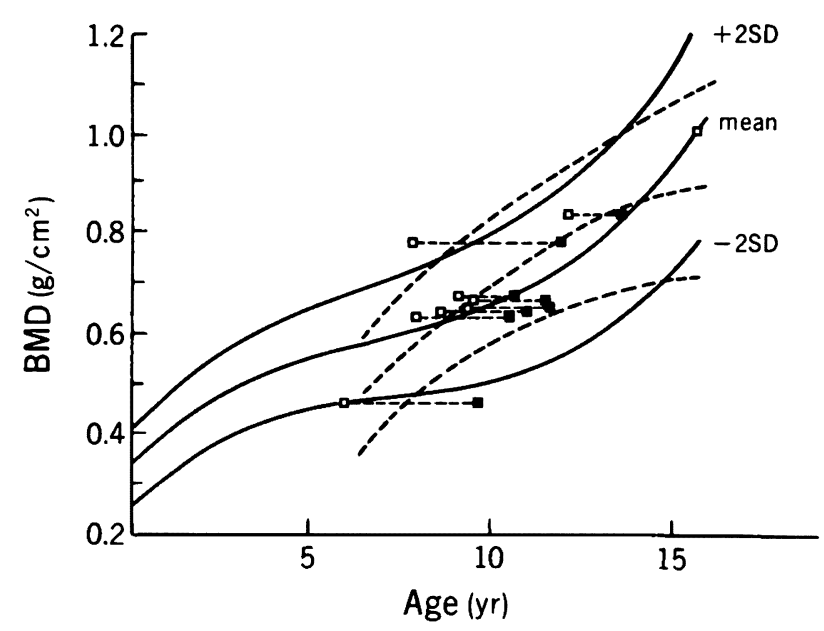

Fig. 1 Relationship between BMD values for the lumbar spine and chronological age (CA) or bone age (BA) in girls with precocious puberty. Clear squares are plotted for CA, and solid squares represent data adjusted to $B A$. Solid lines are standard curves for French children and dotted lines are standard curves for Japanese girls.

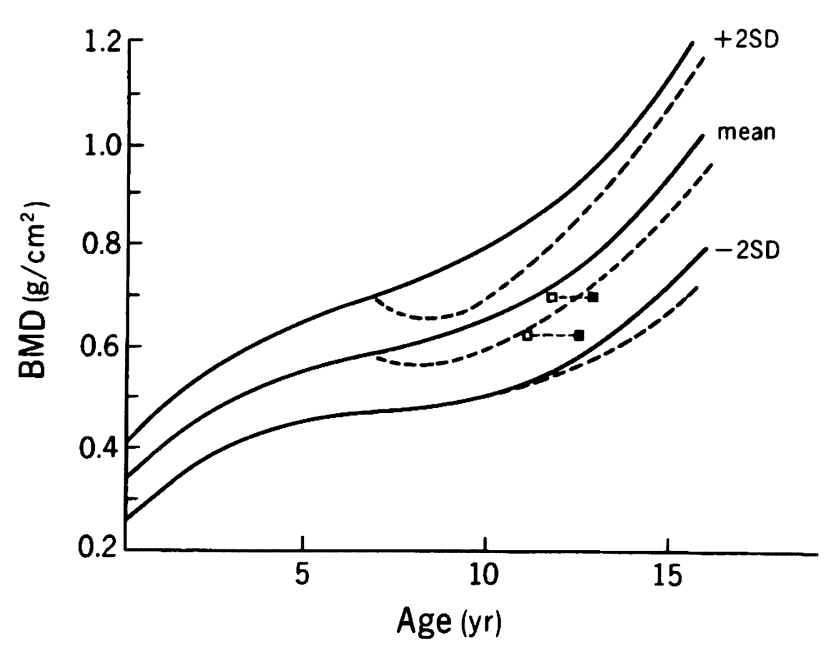

Fig. 2 Relationship between BMD values for the lumbar spine and CA or BA in boys with precocious puberty. Solid lines are standard curves for French children and dotted lines are standard curves for Japanese boys.

\section{Discussion}

In both sexes, a large gain in skeletal mineral content during puberty is necessary to support the increased body weight and muscle strength, and sex steroids play crucial roles in bone growth and mineralization. Recent evidence has shown that estrogen is essential for normal pubertal epiphyseal maturation and skeletal mineralization in both sexes, and that the effect of androgen may be mediated via aromatization to estrogen (4). The present data show that bone maturation (advanced BA) is not associated with BA-matched mineralization in precocious puberty, indicating dissociation between the degree of gained BMD and advanced BA.

It appears that the serum concentration of estrogen and the duration of bone exposure to estrogen account for the present observations of bone status in patients with precocious puberty.

\section{Reffernces}

1. Arisaka O, Arisaka M, Nakayama Y, Yabuta K. Effect of testosterone on bone metabolism in growth hormone-deficient male. Metabolism 1995; 44: 419-23.

2. Glastre C, Braillon P, David L, Cochat P, Meunier PJ, Delmas PD. Measurement of bone mineral content of the lumbar spine by dual energy X-ray absorptiometry in normal children: correlations with growth parameters. J Clin Endocrinol Metab 1990; 70: 1330-3.

3. Sugaya A, Muramatsu Y, Akaji S, Usui N. Spinal bone density in normal Japanese children-analyses by DXA-. (Abstract 166) 
Proc Jpn Soc Bone Mineral Metab 1995:

166.

4. Frank GR. The role of estrogen in pubertal skeletal physiology: epiphyseal maturation and mineralization of the skeleton. Acta Paediatr 1995; 84: 627-30. 